\title{
Hígado: Nuevas Definiciones y Propuestas para SILAT-FCAT-IFAA
}

\author{
Liver: New Definitions and Proposals for SILAT-FCAT-IFAA
}

\author{
César Augusto Durand López ${ }^{1}$; César Ramón Rázuri Bustamante² \& Ademar Cervera Carrillo ${ }^{3}$
}

\begin{abstract}
DURAND, L. C. A.; RÁZURI, B. C. R. \& CERVERA, C. A. Hígado: Nuevas definiciones y propuestas para SILAT-FCAT-IFAA. Int. J. Morphol., 36(2):651-654, 2018.
\end{abstract}

RESUMEN: Presentamos definiciones y propuestas en relación a la anatomía del hígado basados en los resultados de estudiar 286 hígados humanos de ambos sexos, diferentes razas y edades que van desde fetos hasta octogenarios, mediante disección, inyección acrílica, radiología y reconstrucciones tomográficas tridimensionales. Definimos: 1) Segmento portal, 2) Pedículo portal, pedículo segmentario, 3) Fisuras portales, 4) Porta hepática o hilio hepático inferior, 5) Grupos de las venas hepáticas de retorno, 6) Trayecto de la vena hepática izquierda y 7) Conductos biliares aberrantes. Proponemos: 1) Segmento V único formante de la división medial derecha, 2) Siete ramas portales segmentarias terminales para siete segmentos, 3) Irrigación arterial segmentaria, 4) Formación de los conductos biliares, 5) Cambiar la denominación de fisura portal principal por fisura portal intermedia, 6) Incluir dentro del concepto "Fisura umbilical" a la fisura del ligamento redondo y la fisura del ligamento venoso, 7) Fisuras portales horizontales, 8) Venas que drenan en la Cava inferior, 9) No denominar Porción posterior del hígado (A05.8.01.043) al lóbulo caudado.

PALABRAS CLAVE: Hígado; Definiciones y propuestas; SILAT-FCAT-IFAA.

\section{INTRODUCCIÓN}

En los Simposios Ibero-latinoamericanos de Terminología (SILAT) Anatómica, Histológica y Embriológica, se estudia, analiza, traduce y divulga la terminología morfológica internacional, con el fin de que las instituciones educativas de medicina y de otras áreas de la salud, de habla hispana y portuguesa, la empleen cotidianamente.

El Comité Federativo de Terminología Anatómica (FCAT, siglas en inglés de Federative Committee of Anatomical Terminology) es un grupo de expertos que revisan, analizan y discuten los términos morfológicos de las estructuras del cuerpo humano. Dependen de la Federación Internacional de Asociaciones de Anatomistas (IFAA).

La Federación Internacional de Asociaciones de Anatomistas (IFAA del inglés International Federation of Associations of Anatomists) es una organización científica internacional formada por Asociaciones de Anatomistas de todo el mundo que se dedican al estudio de la anatomía humana y las ciencias biomorfológicas.
En relación a la anatomía del hígado, en especial al número de segmentos portales, ha existido gran confusión, ocasionada por la ausencia de definiciones anatómicas.

Luego de estudiar el hígado mediante disecciones, inyecciones acrílicas coloreadas, imágenes radiológicas con contraste, reconstrucciones tomográficas tridimensionales y publicar los resultados, presentamos estas definiciones y propuestas anatómicas.

\section{Definiciones:}

1.- Segmento Portal del hígado: Es una porción de parénquima que presenta tres características: 1) Ser irrigada por una rama terminal de la vena porta hepática (segunda división portal), acompañada de una rama terminal de la arteria hepática propia o su reemplazante, y un conducto de drenaje biliar. 2) Por esta inicial definición no posee anastomosis con los segmentos vecinos, existiendo entre ellos, fisuras portales que los delimitan y aíslan. 3) Todos y cada

\footnotetext{
${ }^{1}$ Cirujano del Hospital de Emergencias "José Casimiro Ulloa” y Profesor de Anatomía Humana, Facultad de Medicina, Universidad Ricardo Palma, Lima-Perú.

${ }^{2}$ Cirujano del Hospital Nacional "2 de Mayo", Profesor de Cirugía en la Universidad Nacional Mayor de San Marcos, Profesor de Anatomía en la Universidad Ricardo Palma. Lima-Perú.

${ }^{3}$ Técnico Anatomista, Universidad Ricardo Palma, Lima-Perú.
} 
uno de los segmentos forman parte del canal parenquimal de la Porta Hepatis (A05.8.01.016), lugar donde reciben su pedículo vascular (Fig. 1) (Durand, 2016, 2017a).

2.- Pedículo portal, pedículo segmentario: Llamado pedículo portal por ser la vena porta hepática (A12.3.12.001) y sus ramas segmentarias las que atraen a las arteriales y biliares, las cuales, no importando su origen, se unen antes de ingresar a su territorio segmentario a través del borde que presentó el segmento para conformar la Porta hepatis (Fig. 1) (Durand, 2016).

3.- Fisuras portales: Planos libres de anastomosis portales, arteriales y biliares entre los segmentos portales, existen tres fisuras verticales: la umbilical (A05.8.01.034) entre los segmentos II-III y el IV, la principal (A05.8.01.035) entre los segmentos IV y V y la derecha (A05.8.01.036) entre los segmentos V y VI-VII; y tres horizontales: entre los segmentos II y III, entre el VI y el VII y entre la cara superior del segmento I y la base de la mitad posterior del IV y el V. El plano de la fisura umbilical se inclina 50 grados a la izquier-

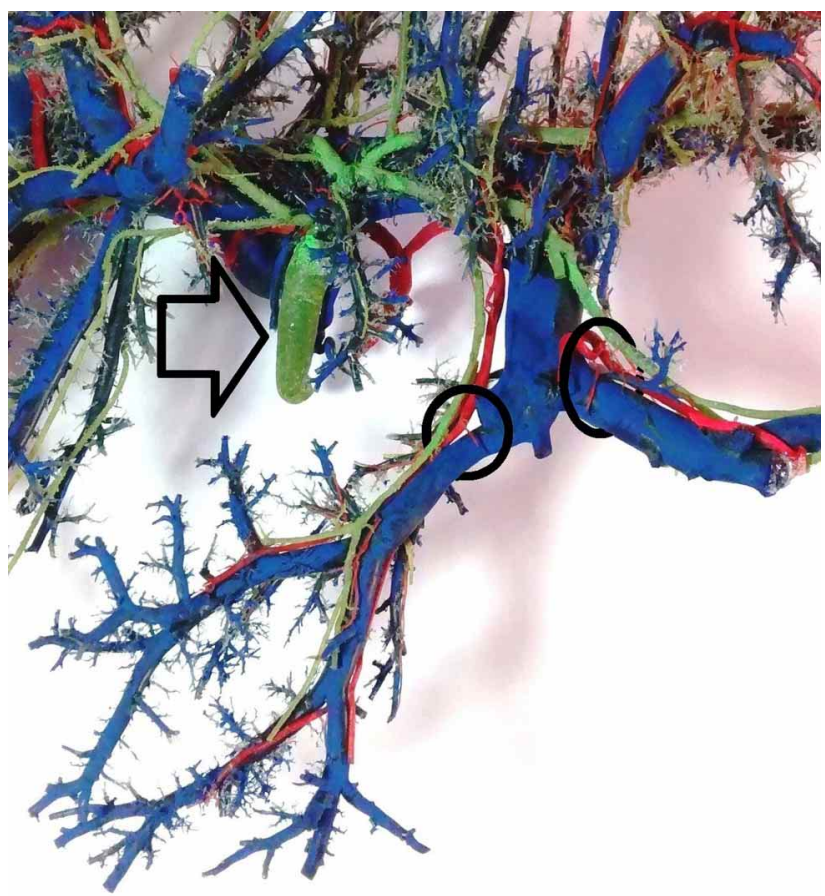

Fig. 1. Acercamiento de vista anterior de acrílico de hígado inyectado en vena porta hepática, arteria hepática propia y conducto hepático común. La flecha señala el pedículo hepático inferior, los círculos rodean los pedículos segmentarios III Y IV que nacen de la parte anterior de la vena porta izquierda en la porta hepatis. Las ramas arteriales y biliares acompañan a la hegemónica vena porta hasta sus ramificaciones más finas. Esta figura grafica las tres características definitorias de un segmento portal: 1) Tener irrigación terminal, 2) Conformar la porta hepatis, 3) Estar aislado de segmentos vecinos por la ausencia de anastomosis (Fisuras portales). da, el plano de la principal 30 grados a la izquierda, y el plano de la derecha 60 grados a la derecha, en relación al plano sagital del hígado (Fig. 2) (Durand, 2017a, 2017b).

\section{4.- Porta hepática o Hilio hepático inferior: Canal} parenquimal formado por todos y cada uno de los segmentos portales en la cara inferior del hígado, en donde el pedículo portal inferior se divide en ramas primarias derecha e izquierda, las que emiten aquí mismo, siete ramas secundarias terminales o segmentarias que luego ingresan a su parénquima respectivo (Fig. 2) (Durand, 2016, 2017a).

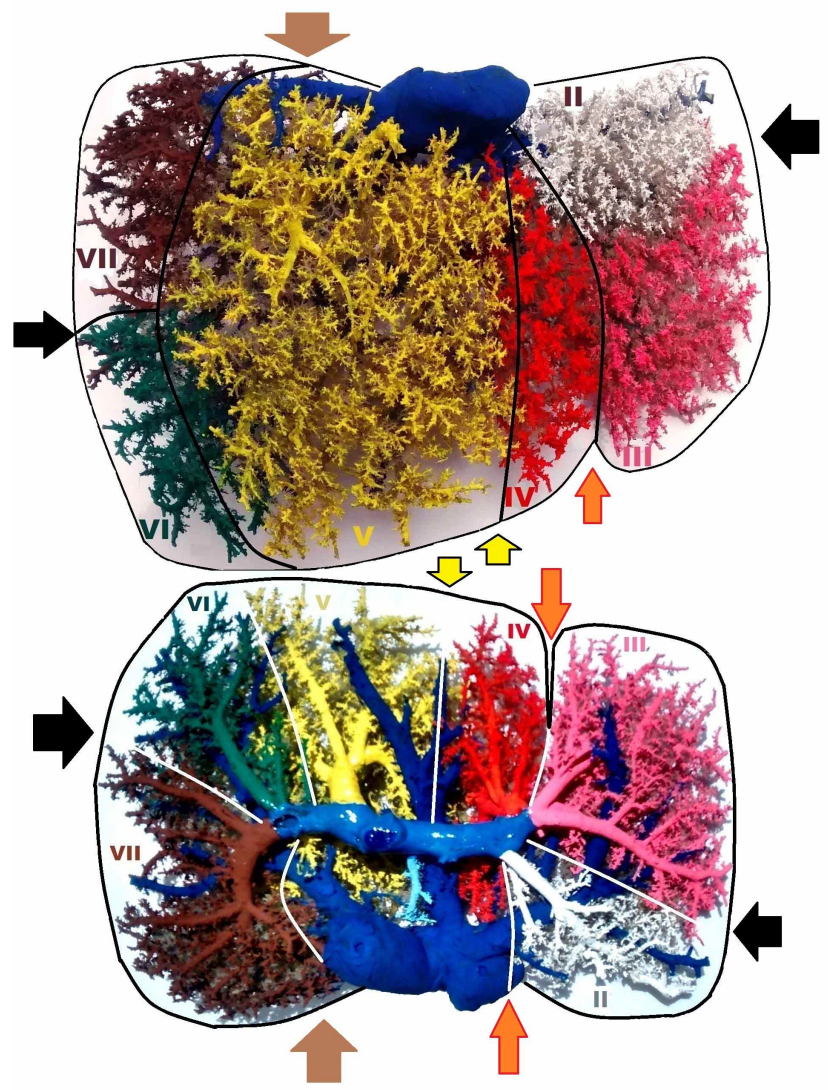

Fig. 2. Hígado inyectado con acrílico en la vena porta hepática y la cava inferior (y por extensión a las venas hepáticas). El segmento I en celeste claro, el II en blanco, el III en rosado, el IV en naranja, el $\mathrm{V}$ en amarillo (entre la fisura principal y la derecha, desde el borde inferior hasta la vena cava inferior solo está presente la rama portal segmentaria V), el VI en verde y el VII en marrón. La cara diafragmática en la imagen superior, la cara visceral en la inferior. La fisura umbilical está señalada por las flechas naranjas, la fisura principal por flechas amarillas, la fisura derecha por flechas marrones; las fisuras horizontales están señaladas por flechas negras. La vena porta en color celeste se ubica en la porta hepatis o hilio inferior del hígado, es aquí donde da siete ramas segmentarias. Observe la vena hepática izquierda naciendo en el centro del segmento III (rosado) y luego cruzar el segmento II (blanco) antes de desembocar en la cava inferior. 
5.- Venas hepáticas (A12.3.09.005), grupo superior e inferior: Las venas hepáticas de retorno se pueden clasificar en dos grupos: Uno superior formado por las venas hepática derecha, intermedia e izquierda; otro inferior formado por las venas hepáticas derechas media e inferior, la intermedia inferior y las venas que drenan el segmento I; todas drenan en la cava inferior (Durand, 2016).

6.- Vena hepática izquierda (A12.3.09.008), trayectoria: Esta vena tiene trayecto intrasegmentario a diferencia de las otras venas hepáticas que tienen trayecto intersegmentario. Tiene tres tipos de conformación y dos tipos de drenaje. En la primera conformación nace en el centro del segmento III, cruza la fisura que lo separa del segmento II, penetra dentro de este segmento y describe una curva de concavidad medial para luego desembocar. La segunda conformación se realiza con el aporte de una vena del segmento II y otra del segmento III. La tercera conformación toma cualquiera de las dos primeras variantes y se le agrega la vena fisural. El primer tipo de drenaje es en la vena hepática intermedia y el segundo en la vena cava inferior. (Fig. 2) (Durand, 2016, 2017b, 2018).

7.- Conductos biliares aberrantes: Son los conductos biliares de los segmentos derechos que no han llegado a desembocar en el conducto derecho y lo hacen directamente en la vesícula biliar o en el conducto cístico, fueron descritos por Hubert von Luschka el año 1863 (Durand, 2016).

\section{Propuestas:}

\section{1.- Segmento $\mathrm{V}$ único formante de la división medial de-} recha (A05.8.01.046): Estudios anatómicos donde usamos técnicas de disección, acrílicos e imágenes reconstituidas de tomografías tridimensionales han demostrado que las ramas segmentarias portales terminales que nacen de la vena Porta derecha e izquierda en la Porta hepatis son siete, el principal aporte consiste en que la división medial derecha (entre la fisura derecha y la principal) posee una sola rama portal segmentaria (Igual que ladivisión medial izquierda), más aún, esta única rama portal $\mathrm{V}$ puede nacer de la porta derecha $(79 \%)$ o de la izquierda (21\%). De esta manera el elemento (A05.8.01.047) debe identificarse como "Segmento V"; la denominación "Segmento medial derecho" sería inexacta cuando el pedículo vascular nazca de la porta izquierda, y para los fines quirúrgicos e intervencionistas lo importante es el origen de esta rama, que la denominaremos con exactitud "rama segmentaria V". El elemento (A05.8.01.048) Segmento VIII nunca fue encontrado, no existe (Fig. 2) (Durand, 2016, 2017).

2.- La terminología anatómica expresa: La rama derecha de la vena porta (A12.3.12.002) se divide en una rama ante- rior (A12.3.12.003) y otra posterior (A12.3.12.004). La rama izquierda (A12.3.12.005) da una rama para el "Lóbulo caudado" (A12.3.12.007), ramas laterales (A12.3.12.009) y ramas mediales (A12.3.12.011).

Debería expresar: En la Porta hepatis, la vena Porta hepática se divide en ramas derecha e izquierda, la izquierda emite ramas segmentarias terminales para los segmentos I, II, III y IV en $79 \%$ de casos, para los segmentos I, II, III, IV y V en $21 \%$; la derecha emite ramas segmentarias V, VI y VII en $79 \%$ y para los segmentos VI y VII en $21 \%$ (IJM 34(45)1522-1530, 2016 Figura 1 y 2, página 1524). El segmento I (La denominación "Lóbulo caudado" no es adecuada ya que es una descripción externa y no vascular) recibe una rama de la porta izquierda en $100 \%$ y además de la porta derecha en $60 \%$ o de la vena porta hepática en $5 \%$ (Durand, 2016, 2018).

3.- La termiología anatómica expresa: La rama derecha de la arteria hepática propia (A12.2.12.030) da la arteria del "lóbulo caudado" (A12.2.12.032), la segmentaria anterior (A12.2.12.033) y la posterior (A12.2.12.034). La rama izquierda (A12.2.12.035) emite la arteria del "lóbulo caudado" (A12.2.12.036), la rama segmentaria medial (A12.2.12.037) y la segmentaria lateral (A12.2.12.038).

Debería expresar: En la Porta hepatis, la arteria hepática propia (en el $70 \%$ de casos es la única que irriga el hígado) (IJM 34(45)1522-1530, 2016, figura 3 página 1524, tabla II, página 1525) se divide en ramas derecha e izquierda, la rama izquierda emite las segmentarias I (30/70 \%), II (70/ $70 \%)$, III (70/70 \%), IV (50/70 \%) y V (1/70 \%), la rama derecha emite la arteria cística y las segmentarias V (69/70 $\%)$, VI (70/70 \%) y VII (70/70 \%) (Durand, 2016, 2018).

4.- La terminología anatómica expresa: El conducto hepático derecho (A05.8.01.062) se forma por la unión de la rama anterior (A05.8.01.063) y la posterior (A05.8.01.064). El conducto hepático izquierdo (A05.8.01.065) se forma por la unión de la rama lateral (A05.8.01.066) y la medial (A05.8.01.067). El conducto de drenaje derecho del "Lóbulo caudado" (A05.8.01.068) y el conducto de drenaje izquierdo del "Lóbulo caudado" (A05.8.01.069). Debería expresar: El conducto hepático derecho está formado por las ramas segmentarias V, VI y VII en $79 \%$ de casos y solo por la rama V en $21 \%$ (En estos casos el tronco VI-VII desemboca en el conducto izquierdo). No existen la rama anterior (A05.8.01.063) ni la posterior (A05.8.01.064). El conducto hepático izquierdo se forma por los troncos y ramas segmentarias I, II, III y IV en $100 \%$ de casos, y se agrega en $21 \%$ el tronco VI-VII (derechos) que desemboca en el hepático izquierdo a $1 \mathrm{~cm}$ del inicio del hepático común. No hay una rama lateral (A05.8.01.066) ni otra medial 
(A05.8.01.067). El drenaje del segmento I (no debe usarse el término "lóbulo caudado") varía de la siguiente manera: En el conducto hepático izquierdo (70 \%), en el conducto hepático derecho (7\%), en ambos conductos (23\%), (Durand, 2016).

\section{5.- Cambiar la denominación "Fisura portal principal" (A05.8.01.035) por "fisura portal intermedia".}

Razonamiento: La fisura portal principal del hígado se denomina así porque divide la porción derecha de la izquierda, sin embargo, cuando la rama portal para el segmento $\mathrm{V}$ nace de la rama portal izquierda ( $21 \%$ de casos), la fisura que divide ambos hemi hígados es la fisura derecha teniendo ahora el rol principal. La fisura principal no se puede llamar fisura sagital porque su plano se inclina 30 grados hacia la izquierda en relación al plano sagital del hígado. Convendría llamarla fisura portal intermedia en concordancia con la vena hepática que contiene, así su nombre e importancia no variaría con el nacimiento de la rama portal segmentaria V (Durand, 2017b, 2018).

6.- Incluir dentro del concepto "Fisura umbilical" a la fisura del ligamento redondo y la fisura del ligamento venoso.

Razonamiento: El canal parenquimal que forma el borde inferior del plano de la fisura umbilical contiene de delante hacia atrás al ligamento redondo, la porción umbilical de la vena porta izquierda y al ligamento venoso, es redundante llamar fisura del ligamento redondo (A05.8.01.014) o fisura del ligamento venoso (A05.8.01.010) a porciones del canal parenquimal del borde inferior de la fisura umbilical (Durand, 2016, 2018).

7.- Fisuras portales horizontales: Como lo indica su nombre tienen sentido horizontal, no tienen contenido vascular, separan el segmento II del III, el VI del VII y la cara superior del I con la base de la mitad posterior de los segmentos IV y $\mathrm{V}$.

8.- Venas que drenan en la Cava inferior: Agregar las venas hepáticas inferiores a la lista de venas que drenan en la cava inferior.

9.- No denominar "Porción posterior del hígado" (A05.8.01.043) al lóbulo caudado. Razonamiento: La porción hepática es un concepto vascular originado por la división del pedículo vascular inferior del hígado (vena porta hepática, arteria hepática propia) en rama derecha para la porción derecha y rama izquierda para la porción izquierda, el segmento portal I (lóbulo caudado) que es también un concepto vascular recibe su rama segmentaria portal de la porta izquierda en $100 \%$ de casos, es decir forma parte de la por- ción izquierda del hígado. El concepto "Lóbulo caudado" es descriptivo de la superficie hepática, no es un concepto vascular.

DURAND, L. C. A.; RÁZURI, B. C. R. \& CERVERA, C. A. Liver: New definitions and proposals for SILAT-FCAT-IFAA. Int. J. Morphol., 36(2):651-654, 2018.

SUMMARY: We present definitions and proposals in relation to the anatomy of the liver based on our investigation using dissection, acrylic injection, tomographic and radiological studies of 286 human livers of sexes, different races and ages ranging from fetuses to octogenarians. We define: 1) Portal segment, 2) Portal pedicle, segmental pedicle, 3) Portal fissures, 4) Porta Hepatis or Lower hepatic hilum, 5) Groups of hepatic return veins, 6) Left hepatic vein pathway and 7) Aberrant bile duct. We propose: 1) Segment V only formant of the right medial division, 2) Seven terminal segmental portal branches, 3) Segmental arterial irrigation, 4) Formation of bile ducts, 5) Change the denomination of main portal fissure by intermediate portal fissure ,6) Include within the concept "umbilical fissure" the fissure of the round ligament and fissure of the venous ligament, 7) Horizontal portal fissures, 8) Veins that drain in the inferior vena Cava, 9) Do not call the posterior portion of the liver (A05.8.01.043) to the caudate lobe.

KEY WORDS: Liver; Definitions and proposals; SILAT-FCAT-IFAA.

\section{REFERENCIAS BIBLIOGRÁFICAS}

Durand, L. C. A. Liver: seven hepatic segments. Int. J. Morphol., 34(4):1522-30, 2016.

Durand, L. C. A. Liver: the portal segment V. Int. J. Morphol., 35(1):3637, 2017.

Durand, L. C. A.; Torres, G. C. A. \& Cervera, C. A. Hepatic portal segmentation using reconstructed three-dimensional tomography images. Int. J. Morphol., 35(3):884-8, 2017.

Durand, L. C. A. Left part of the liver (A05.8.01.037). Int. J. Morphol., 36(1):113-20, 2018

Dirección para correspondencia

Dr. César Augusto Durand López

Hospital Emergencias

Av. Roosevelt 6355

Miraflores

Lima

PERÚ

Email: cesaraugustoo1@gmail.com

Recibido : 13-11-2017

Aceptado: 04-02-2018 